\title{
Estudio de los factores determinantes para el desarrollo de sensores SERS efectivos y accesibles
}

\section{Study of the determinants for the development of effective and accessible SERS sensors}

\author{
Yulán Hernández ${ }^{1}$, Angeline Saldaña', Jorge Sifuentes', Betty Galarreta' \\ ${ }^{1}$ Pontificia Universidad Católica del Perú, Departamento de Ciencias-Sección Química, Lima, Perú \\ Recibido (Recieved): 16/10/2018 \\ Aceptado (Accepted): 11/12/2018
}

\section{RESUMEN}

La nanotecnología ha aportado a la ciencia un sinfín de herramientas para la mejora de los sistemas de detección, como es el caso de la espectroscopía Raman amplificada en superficie (SERS), que aumenta en hasta 8-10 órdenes de magnitud las señales obtenidas mediante la técnica tradicional. Sin embargo, su éxito depende del material y la morfología de las nanopartículas, así como de la adsorción de los analitos sobre las mismas, aspectos que no siempre se tienen en cuenta a la hora de diseñar sensores. En este trabajo se ha explorado la influencia de estos factores mediante el uso de una molécula modelo, el azul de metileno (MB), y dos metabolitos del café, trigonelina y ácidos clorogénicos (CGAs), potenciales marcadores de calidad de este producto agropecuario tan importante en el Perú. Así, se ha determinado una mayor amplificación de las señales al emplear nanoestructuras de plata versus oro, debido a su mayor poder de dispersión, así como para triángulos versus esferas, por la distribución no homogénea del campo electromagnético. Además, al analizar los dos metabolitos seleccionados, se ha observado como sólo en uno de los dos casos se produce un aumento de las señales respectivas, lo cual demuestra que una adecuada interacción entre la molécula y el metal es esencial para evitar la limitación de algunos modos vibracionales. Por último, en base a los resultados anteriores, se ha desarrollado un sensor rápido, sencillo y portátil para la cuantificación de trigonelina en solución, que podría ser implementado en control de calidad de productos agropecuarios como el café.

Palabras Clave: SERS, nanopartículas, oro, plata, metabolitos, café

\section{ABSTRACT}

Nanotechnology has provided endless tools to improve detection systems in the most diverse fields of science. One of those tools is the development of surface enhanced Raman spectroscopy (SERS) that promotes the amplification in up to 8-10 orders of magnitude of the conventional Raman signals. However, the success of this analytical technique depends on the core material of the nanoparticles, their morphology and the adsorption pattern of the analytes on the metal surface, aspects that are not always considered when designing this type of sensors. This work has focused on the effect of these features using a reference molecule such as methylene blue (MB) and two coffee metabolites, trigonelline and chlorogenic acids (CGAs), known as potential quality markers of this agricultural product so important in Peru. Herein, we report higher Raman signals when using silver nanostructures over their gold homologues, due to their higher scattering cross-section, and nanotriangles over nanospheres, due to the non-homogeneous distribution of the electromagnetic field. In addition, when the two coffee metabolites were evaluated, only one of them induced an increase of its respective signals, which proves the relevance of the interaction between the analysed molecule and the metal to not hinder some of its vibrational modes. Lastly, these results have been taken into account for the development of a quick, easy and portable sensor for the quantification of trigonelline in solution, which could be implemented in the quality control of some important agricultural products such as coffee.

Keywords: SERS, nanoparticles, gold, silver, metabolites, coffee

\section{INTRODUCCION}

Desde que en 1959 Richard Feynman anunciara la gran importancia que adquirirían los materiales cuyas dimensiones fueran "un millón de veces más pequeñas que la cabeza de un alfiler" (1), y con el desarrollo de técnicas como la microscopía de efecto túnel (STM) en los años 80, la nanociencia y sus potenciales aplicaciones no han parado de crecer. Todo ello se debe a que estos materiales presentan una serie de propiedades ópticas, magnéticas, electroquímicas, etc. muy distintas a las de sus homólogos a nivel macroscópico, precisamente por sus minúsculas dimensiones, generalmente acotadas a 1-100 nm (2,3).

Una de las ramas que se ha visto más favorecida con la gran expansión de los nanomateriales, es el

\footnotetext{
${ }^{*}$ Corresponding author

E-mail: yhernandez@pucp.pe
} 
desarrollo de sensores, ya que la versatilidad de estos sistemas en combinación con las nuevas propiedades que presentan, ha fomentado la mejora de algunas técnicas analíticas tradicionales y la aparición de otras nuevas, lo que ha permitido disminuir de manera muy notable los límites de detección, manteniendo muy buenos valores de selectividad. Una de las técnicas que más favorecida se ha visto es la espectroscopía Raman, ya que se trata de una técnica muy selectiva pero cuya baja sensibilidad ha limitado mucho su aplicación (4). Sin embargo, en 1974 Fleischmann (5) descubrió que al llevar a cabo el análisis de piridina sobre una superficie de plata, las señales Raman de la misma sufrían una gran amplificación. Tres años más tarde Albrecht (6) y Van Duyne (7) consiguieron interpretar correctamente el fenómeno observado, lo cual se atribuye a las excepcionales propiedades ópticas que presentan las nanoestructuras de oro y plata. Estos estudios han dado lugar a lo que se conoce como espectroscopía Raman amplificada en superficie o SERS (por sus siglas en inglés).

Las nanopartículas de metales nobles como el oro (AuNPs) y la plata (AgNPs), presentan unas intensas bandas de extinción que se conocen como bandas de plasmón superficial localizado (LSPR) (8). El origen de estas se encuentra en las oscilaciones de la carga electrónica que se dan en la superficie del metal, de tal forma que al ser irradiado a la frecuencia de resonancia, se produce un aumento del campo electromagnético. La especial relevancia en el caso de AuNPs y AgNPs es que la longitud de onda a la que aparecen estas bandas decae en la zona del visible-infrarrojo cercano, lo que aumenta su aplicabilidad y es la responsable de los colores tan intensos que presentan. Además, la frecuencia de resonancia y la magnitud de la contribución de absorción o dispersión dependen de otros parámetros como el material, el tamaño, la forma y el entorno de las nanopartículas, por lo que es posible seleccionar las nanoestructuras más adecuadas para cada aplicación concreta.

Aunque la mayoría de trabajos utilizan nanopartículas esféricas, dado que se tratan de estructuras isotrópicas, el campo electromagnético inducido en la partícula es uniforme a lo largo de toda ella. En muchos de estos trabajos se aumenta la fuerza iónica del medio para inducir la agregación de las nanopartículas, ya que al reducir la distancia entre ellas, se produce un solapamiento de los campos electromagnéticos en esas zonas, denominadas hot spots, y en las cuales el incremento de las señales Raman alcanza valores máximos (9). Sin embargo, en el caso de nanopartículas anisotrópicas, como los triángulos, su inhomogeneidad en el espacio, provoca la desigual distribución del campo electromagnético en la superficie de la estructura, haciendo que existan hot spots que amplifiquen las señales sin necesidad de requerir un fenómeno tan poco controlado como es la agregación de los sistemas.

doi: https://doi.org/10.21754/tecnia.v28i2.560
Por otra parte, cabe destacar que se habla de dos factores que contribuyen al aumento de las señales Raman, por una parte el electromagnético ya explicado, y por otro, la transferencia electrónica que se puede producir entre la superficie del metal y la molécula estudiada, siendo el más influyente el primer factor (10). De hecho, según cómo se produzca la interacción analito-metal los cambios espectrales pueden ser muy notables, no sólo por efecto de la transferencia electrónica y aumento de las señales, sino porque algunos modos vibracionales pueden verse restringidos, lo cual tiene un efecto negativo en el proceso, viendo una reducción de la señal correspondiente.

Por estos motivos, para este trabajo se han seleccionado nanoesferas de plata (AgNS) y de oro (AuNS) y nanotriángulos de oro (AuNT), a fin de evaluar tanto el material como la morfología frente a las señales SERS de una molécula modelo como es el azul de metileno (MB). Asimismo, se ha estudiado la respuesta obtenida para dos metabolitos de café, trigonelina y ácidos clorogénicos, para ver la influencia de la interacción analito-nanopartícula puede llegar a tener en el aumento de las señales Raman. Finalmente, en base a estos resultados, se ha desarrollado un sensor de cuantificación de trigonelina, como potencial marcador de calidad de un producto tan importante a nivel mundial como el café.

\section{MATERIALES Y MÉTODOS}

Todos los materiales fueron adquiridos en Sigma Aldrich (St Louis, USA) y todas las soluciones se prepararon con agua MilliQ.

\subsection{Síntesis de AuNS}

Se sintetizaron AuNS de $14 \pm 1 \mathrm{~nm}$ de acuerdo a lo descrito por Turkevich (11) y Frens (12), mediante la reducción de una sal de oro con citrato sódico. Brevemente, en un balón se añaden $508 \mu \mathrm{L}$ de $\mathrm{HAuCl}_{4}$ $50 \mathrm{mM}$ y $24 \mathrm{~mL}$ de agua, y se calienta la mezcla bajo reflujo y en agitación constante. Una vez en ebullición, se añade $1 \mathrm{~mL}$ de citrato sódico $100 \mathrm{mM}$ y se deja en agitación durante 30 minutos.

\subsection{Síntesis de AgNS}

De manera análoga se prepararon AgNS de $40 \pm 7 \mathrm{~nm}$ según el método reportado por Lee-Meisel (13). Para ello, se calienta una solución $1 \mathrm{mM}$ de $\mathrm{AgNO}_{3}$ bajo reflujo y en agitación constante, y al ebullir se añade $1 \mathrm{~mL}$ de citrato sódico dihidratado $34 \mathrm{mM}$. Tras 1 hora en ebullición, la mezcla se lleva a temperatura ambiente.

\subsection{Síntesis y funcionalización de AuNT}

La síntesis de AuNT de 52ะ16 nm de lado se llevó a cabo mediante el método de Pelaz et al. (14) con ligeras modificaciones. En nuestro caso, se añaden $8.25 \mathrm{~mL}$ de $\mathrm{Na}_{2} \mathrm{~S}_{2} \mathrm{O}_{3} 0.5 \mathrm{mM}$ con una bomba de jeringa (flujo 9 
$\mathrm{mL} / \mathrm{min}$ ) sobre $5 \mathrm{~mL}$ de una solución de $\mathrm{HAuCl}_{4} 2 \mathrm{mM}$, manteniendo una temperatura de $18-19^{\circ} \mathrm{C}$. Tras 9 minutos en agitación constante, se añade $1 \mathrm{~mL}$ de la solución de $\mathrm{Na}_{2} \mathrm{~S}_{2} \mathrm{O}_{3}$ y se deja en agitación durante 2 horas.

Para la determinación de trigonelina, los AuNTs fueron funcionalizados con ácido mercaptopropiónico (MPA) $46 \mu \mathrm{M}$ en presencia de $\mathrm{NaOH} 4 \mathrm{mM}$. Tras 16 horas en agitación, se eliminó el exceso de MPA por centrifugación (AuNT@MPA).

\subsection{Caracterización de las nanopartículas}

Todas las nanopartículas preparadas fueron caracterizadas mediante espectroscopía UV-Vis-NIR (espectrofotómetro Genesys 10S, Thermo ScientificUSA) y microscopía electrónica de transmisión (microscopio LVEM5, Delong Instruments-República Checa).

\subsection{Análisis mediante SERS}

Los análisis SERS con MB se llevaron a cabo utilizando dos espectrómetros Raman portátil (iRaman Plus, B\&WTek - USA) con fuentes de excitación a 532 $\mathrm{nm}$ y a $785 \mathrm{~nm}$. En el caso de la fuente a $532 \mathrm{~nm}$ (10 s, 10 adq, $37 \mathrm{~mW}$ de potencia), se usó en combinación con un microscopio y un objetivo 40x, depositando las muestras (sin tratar después de la síntesis) sobre un portamuestras convencional de vidrio y secándolas sobre una placa calefactora. Con la fuente de $785 \mathrm{~nm}$ (1 s AuNS y AuNT/100 ms AgNS, 10 adq, $277 \mathrm{~mW}$ de potencia), los análisis se llevaron a cabo en solución y se utilizó un portacubetas proporcionado por la misma marca comercial. Salvo que se indique lo contrario, se diluyeron las nanopartículas 1:5, teniendo en cuenta que la concentración de AgNS es $1 \mathrm{nM}$ (15), de AuNS $12 \mathrm{nM}$ (16) y de AuNT se estima en 0,8 nM (asumiendo que todo el oro se ha reducido y que el rendimiento es aprox. 70\%) $(14,17)$.

En el caso de los análisis finales de trigonelina, los ensayos se llevaron a cabo en solución, haciendo diluciones 1:5 de AuNT@MPA y utilizando la fuente de $785 \mathrm{~nm}$ ( $2 \mathrm{~s}, 10 \mathrm{adq}, 110.8 \mathrm{~mW}$ ). Los espectros fueron estudiados con OriginPro 2019, analizando la intensidad máxima entre $1005-1045 \mathrm{~cm}^{-1}$ (línea base por el método de suavizado asimétrico de mínimos cuadrados).

\section{RESULTADOS Y DISCUSIÓN}

Se procedió a la preparación de los tres tipos de nanopartículas seleccionados mediante los métodos anteriormente descritos.

En el caso de los AuNT, las condiciones de reacción fueron optimizadas para aumentar el rendimiento de nanotriángulos cuya banda plasmónica se encuentra centrada en $780 \pm 30 \mathrm{~nm}$.

En la figura 1 se puede apreciar la caracterización de todas las nanopartículas mediante espectroscopía UVVis-NIR y microscopía electrónica de transmisión (TEM).

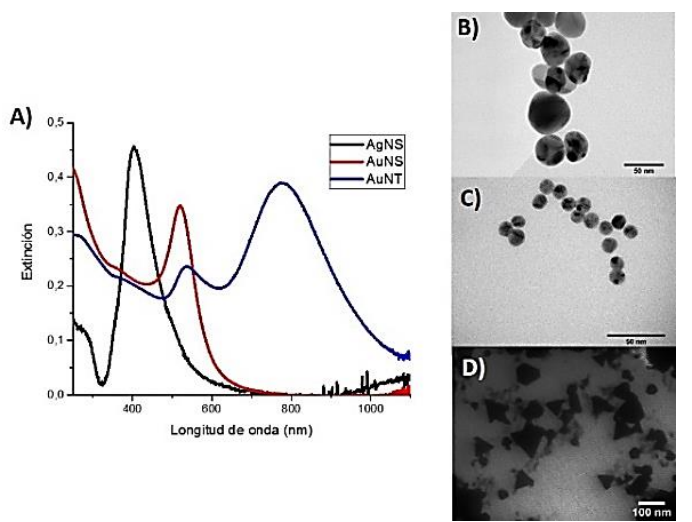

Figura 1. A) Espectro UV-Vis-NIR de las nanopartículas preparadas, y micrografías TEM de B) AgNS, C) AuNS y D) AuNT. Las escalas representan $B$ ) y C) $50 \mathrm{~nm}$ y D) $100 \mathrm{~nm}$.

3.1 Análisis de la influencia del material y la forma en SERS con azul de metileno (MB)

Una vez obtenidas las nanoestructuras se procedió a su análisis en presencia de MB como molécula modelo mediante espectroscopía SERS. En primer lugar, se utilizó la fuente de $532 \mathrm{~nm}$ acoplada a un microscopio, para ello se depositaron las nanopartículas correspondientes y después $3 \mu \mathrm{L}$ de una solución $10 \mathrm{mM}$ de MB. Tal y como se observa en la figura $2 \mathrm{~A}$, las AgNS fueron las que indujeron un mayor aumento de las señales Raman seguidas por los AuNT y en último lugar las AuNS. De estos resultados, cabe destacar que la plata induce un mayor aumento de las señales, lo cual se explica por su mayor poder de dispersión de la luz, que fomenta un mayor aumento en el campo electromagnético localizado. Por otra parte, en cuanto a la forma, los AuNT proporcionaron ligeramente mejores resultados que las AuNS, debido a la presencia de hot spots sin necesidad de inducir agregación en el sistema.

Estos resultados fueron confirmados en solución mediante el uso de la fuente Raman a $785 \mathrm{~nm}$, ya que los resultados obtenidos fueron análogos a los descritos anteriormente (figura 2B-D). Se probaron dos concentraciones distintas, 1 y $100 \mu \mathrm{M}$, confirmando lo descrito hasta el momento. Cabe destacar que a la vista de los resultados obtenidos para ambas estructuras de oro y teniendo en cuenta que la concentración de los AuNT se estima 15 veces menor que la de AuNS, se puede determinar que los primeros proporcionaron de nuevo mejores resultados lo que vuelve a demostrar la mayor efectividad de las nanoestructuras anisotrópicas como amplificadoras de la señal.

3.2 Análisis de la influencia de la adsorción con trigonelina y ácidos clorogénicos (CGAs)

Una vez establecidos los parámetros más importantes, se decidió probar el efecto de la interacción analito-nanopartícula para el aumento de las señales Raman. Para ello, se decidió implementar los 


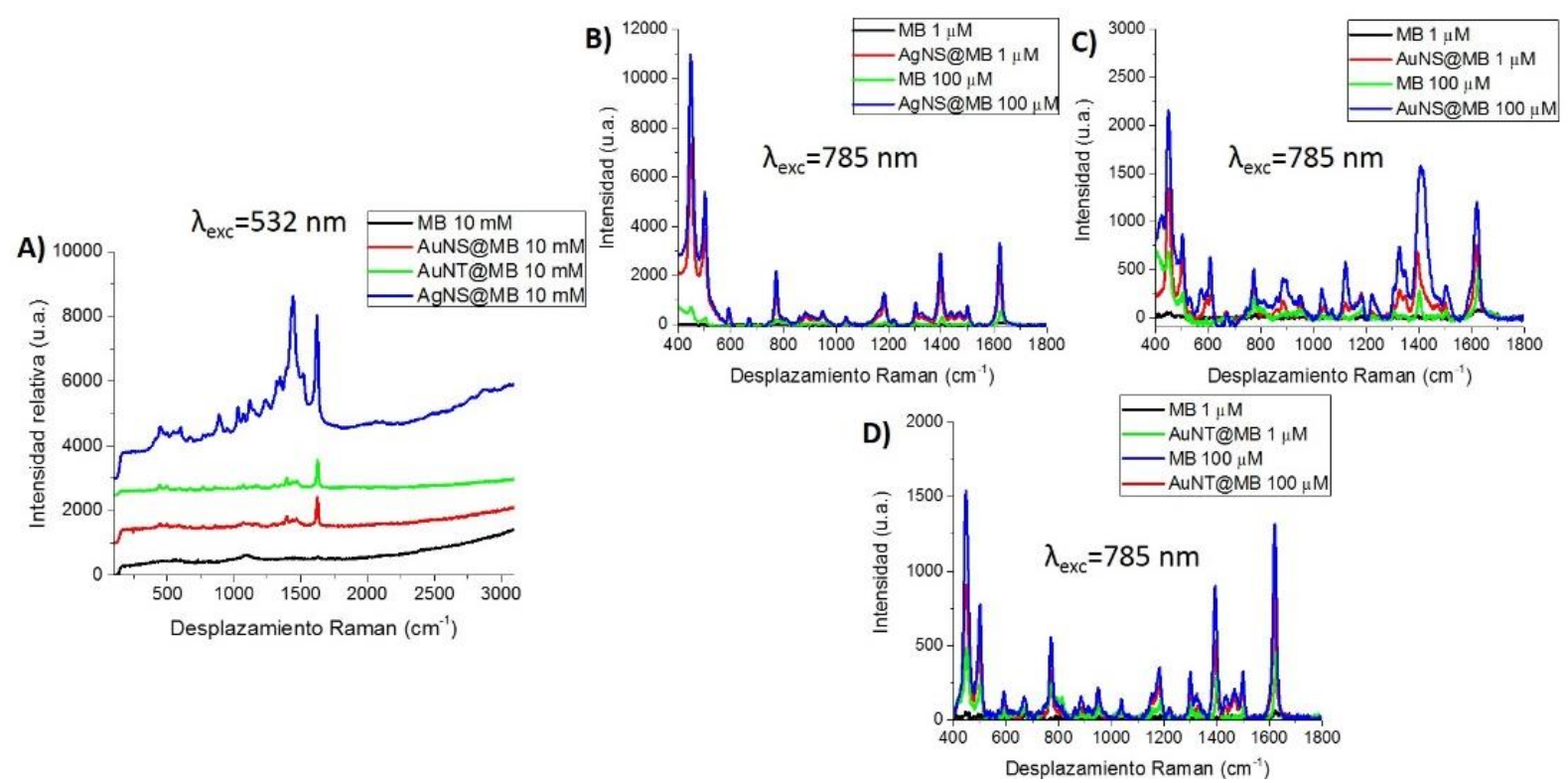

Figura 2. Espectros Raman de MB en presencia y ausencia de A) diversos tipos de nanopartículas medido con fuente de $532 \mathrm{~nm}$; y B) AgNS, C) AuNS y D) AuNT, medido con fuente de $785 \mathrm{~nm}$.

TABLA 1. Bandas de dispersión Raman de trigonelina (18) y CGAs (19) y sus correspondientes modos vibracionales.

\begin{tabular}{|c|c|c|}
\hline & Trigonelina & CGAs \\
\hline Modo vibracional $^{\mathrm{a}}$ & $\begin{array}{c}\text { Desplazamiento } \\
\left(\mathrm{cm}^{-1}\right)\end{array}$ & $\begin{array}{c}\text { Desplazamiento } \\
\left(\mathrm{cm}^{-1}\right)\end{array}$ \\
\hline $\begin{array}{c}\beta\left(\mathrm{CO}_{2}\right), \rho\left(\mathrm{C}_{\text {anillo- }^{-}}\right. \\
\left.\mathrm{C}_{\text {carboxilo }}\right)\end{array}$ & 515 & \\
\hline $\begin{array}{l}\rho(\mathrm{CO}), \beta(\mathrm{COH}), \\
\mathrm{v}\left(\mathrm{C}_{\text {anillo }} \mathrm{C}_{\text {carboxilo }}\right)\end{array}$ & 757 & \\
\hline $\mathrm{v}\left(\mathrm{C}_{\text {metil }}-\mathrm{N}\right)$ & 800 & \\
\hline Fen. $[\omega(\mathrm{CH})]$ & & 815 \\
\hline Fen. $[\omega(\mathrm{CH})]$ & & 847 \\
\hline Cicl. $[u(C O)]$ & & 856 \\
\hline Cicl. $[v(C O)]$ & & 970 \\
\hline Cicl. $[v(C O)]$ & & 980 \\
\hline $\begin{array}{l}\mathrm{v}(\mathrm{C}-\mathrm{C})_{\text {anillo }} \\
\mathrm{v}(\mathrm{C}-\mathrm{N})_{\text {anillo }}\end{array}$ & 1034 & \\
\hline Cicl. $\left[\delta_{\text {anillo }}\right]$ & & 1119 \\
\hline Fen. $[\delta(\mathrm{CH})]$ & & 1167 \\
\hline Fen. $[\delta(\mathrm{C} \mathrm{CH})]$ & & 1206 \\
\hline Fen. $[v(C O)]$ & & 1244 \\
\hline Fen. $[\rho(\mathrm{CH})]$ & & 1260 \\
\hline Fen. $[\rho(\mathrm{CH})]$ & & 1277 \\
\hline Fen. $[\delta(\mathrm{OH})]$ & & 1285 \\
\hline Fen. $\left[\delta_{\text {anillo }}\right]$ & & 1342 \\
\hline Cicl. $[\omega(\mathrm{CH})]$ & & 1367 \\
\hline $\begin{array}{c}\mathrm{v}_{\text {as }}(\mathrm{COO}-), \\
\mathrm{v}\left(\mathrm{C}_{\text {anillo }} \mathrm{C}_{\text {carboxilo }}\right), \\
\rho\left(\mathrm{CO}_{2}\right)\end{array}$ & $1384 \mathrm{w}$ & \\
\hline Fen. $\left[\nu_{\text {anillo }}\right]$ & & 1444 \\
\hline $\begin{array}{l}\mathrm{v}(\mathrm{C}-\mathrm{C})_{\text {anillo, }} \\
\mathrm{v}(\mathrm{C}-\mathrm{N})_{\text {anillo }}\end{array}$ & 1597,1643 & \\
\hline Fen. $\left[v_{\text {anillo }}\right]$ & & 1605 \\
\hline Et. $[v(C=C)]$ & & 1631 \\
\hline $\mathrm{v}(\mathrm{C}=\mathrm{O})$ & 1726 & \\
\hline
\end{tabular}

${ }^{a}$ v-estiramiento, Vas-estiramiento simétrico, $\delta$-flexión, $\rho$ balanceo, $\beta$-flexión en el plano, $\omega$-aleteo.

doi: https://doi.org/10.21754/tecnia.v28i2.560 resultados anteriores en la detección de conocidos metabolitos de café, como trigonelina y CGAs. De esta forma, se pretendía evaluar que no todas las moléculas pueden ser analizadas mediante un mismo sistema debido a la influencia de la adsorción específica de cada analito. En primer lugar, se analizaron los espectros Raman de cada una de las especies por separado para obtener las bandas más características en cada caso (Tabla 1).
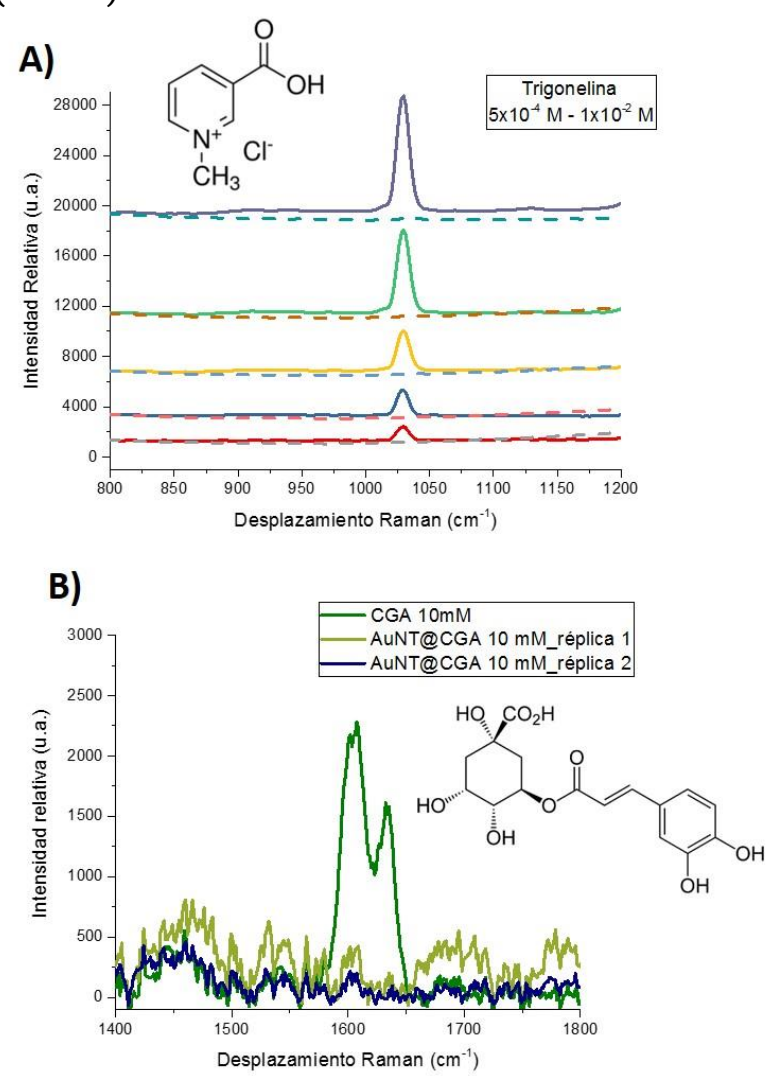

Figura 3. Espectros Raman en presencia (línea sólida) y ausencia (línea punteada) de AuNT para A) distintas concentraciones de trigonelina y B) CGAs $10 \mathrm{mM}$.

Revista TECNIA Vol. 28 Nº 1 Agosto - Diciembre 2018 
Llegados a este punto se analizaron diversas concentraciones de los metabolitos en presencia de AuNT (diluidos 1:10 tras la síntesis). Como se puede apreciar en la figura $3 \mathrm{~A}$, el aumento de la señal a $1034 \mathrm{~cm}^{-1}$ de la trigonelina es muy claro, permitiendo llegar a detectar hasta $500 \mu \mathrm{M}$ del metabolito. Por el contrario, tal y como se ve en la figura $3 \mathrm{~B}$, en el caso de Ios CGAs el efecto es el contrario, ya que las bandas más intensas (1605 y $1631 \mathrm{~cm}^{-1}$ ), correspondientes al $v_{\text {anillo }}$ del grupo fenilo y a $v(C=C)$ del grupo etilo, ven reducida su intensidad hasta llegar a desaparecer. Este efecto se debe a que la adsorción de los CGAs sobre los AuNT se produce a través del grupo fenilo, lo que limita la vibración del mismo, haciendo que su señal decaiga hasta casi desaparecer. Así se demuestra, la gran importancia de la adsorción de las moléculas a la hora de diseñar sensores SERS.

\subsection{Cuantificación de trigonelina}

Una vez determinados los parámetros fundamentales para una cuantificación efectiva, se procedió al desarrollo de un sensor para trigonelina, un metabolito del café seleccionado como potencial marcador de calidad de dicho producto (18). Para maximizar la adsorción de la trigonelina sobre AuNT, estos se funcionalizaron con MPA (AuNT@MPA) para favorecer la interacción electrostática entre ambas especies. Al analizar distintas concentraciones de trigonelina, se pudo establecer una buena relación lineal entre la concentración observada y la intensidad de la señal observada a $1034 \mathrm{~cm}^{-1}$ (figura 4) llegando a un LOD de 0,122 $\mathrm{mM}$ (calculado siguiendo el método de propagación de errores propuesto por Long et al.(20)), mediante un sencillo y rápido ensayo en solución.

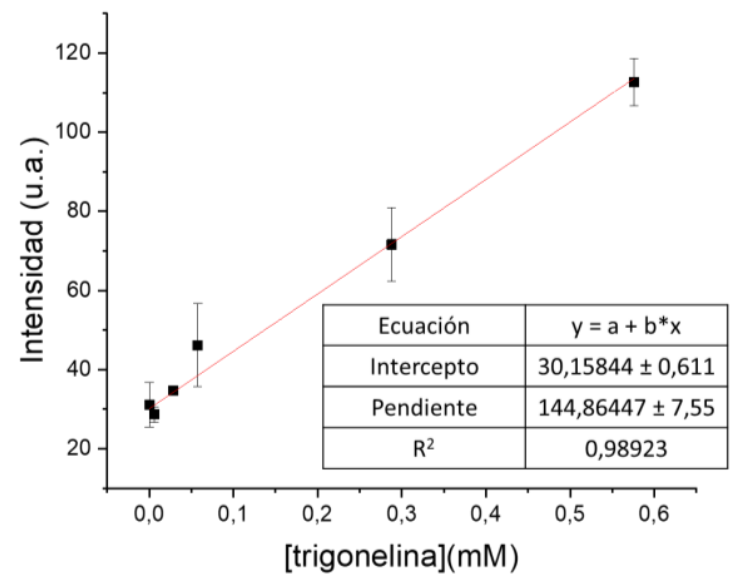

Figura 4. Curva de calibración de trigonelina en solución utilizando AuNT@MPA y espectroscopía SERS. Cada punto representa el promedio de un mínimo de 2 medidas independientes.

\section{CONCLUSIONES}

Mediante la preparación de distintas nanopartículas de oro y plata y su aplicación en SERS con distintas moléculas, se ha podido determinar la gran influencia del material utilizado y la forma de las nanoestructuras doi: https://doi.org/10.21754/tecnia.v28i2.560 en el aumento de las señales. Así, la plata ha demostrado inducir mayores factores de aumento debido a su mayor poder de dispersión, mientras que el uso de nanopartículas anisotrópicas también mejora las señales debido a la concentración del campo electromagnético en determinadas zonas.

Además, se ha demostrado, que no sólo son importantes estos factores para el éxito final de un sensor SERS, sino que la adsorción de cada molécula a analizar también es de gran importancia y, según el tipo de interacción que presente con el metal, las señales pueden verse amplificadas o inhibidas.

Por último, se ha utilizado toda esta información para el desarrollo de un sensor SERS de trigonelina en solución, que podría ser implementado en la industria del café. De esta forma, se dan las claves para el desarrollo de sensores rápidos y selectivos, mediante una técnica portátil y que no precisa de exhaustivos tratamientos previos. Todo ello puede contribuir a la mejora de los controles de calidad de productos agropecuarios.

\section{AGRADECIMIENTOS}

Este trabajo fue financiado por la Dirección de Gestión de la Investigación de la PUCP a través de las subvenciones DGI-2016-352 y DGI-2017-482.

\section{REFERENCIAS}

[1] Richard P. Feynman. 1960. There's plenty of room at the bottom. Eng Sci. 23(5):22-36.

[2] El-Sayed MA. 2001. Some interesting properties of etals confined in time and nanometer space of different shapes. Acc Chem Res. 34(4):257-64.

[3] Whitesides GM. 2003. The "right" size in nanobiotechnology. Nat Biotechnol. 21(10):1161-5. [4] Cialla D, März A, Böhme R, Theil F, Weber K, Schmitt M, et al. 2012. Surface-enhanced Raman spectroscopy (SERS): Progress and trends. Anal Bioanal Chem. 403(1):27-54.

[5] Fleischmann M, Hendra PJ, McQuillan AJ. 1974.

Raman spectra of pyridine adsorbed at a silver electrode. Chem Phys Lett. 26(2):163-6. [6] Albrecht MG, Creighton JA. 1977. Anomalously Intense Raman Spectra of Pyridine at a Silver Electrode. J Am Chem Soc. 99(15):5215-7.

[7] Jeanmaire DL, Van Duyne RP. 1977. Surface Raman spectroelectrochemistry: Part I. Heterocyclic, aromatic, and aliphatic amines adsorbed on the anodized silver electrode. J Electroanal Chem. 84(1):1-20.

[8] Mayer KM, Hafner JH. 2011. Localized surface plasmon resonance sensors. Chem Rev. 111(6):3828-57. [9] Kleinman SL, Frontiera RR, Henry A-I, Dieringer JA, Van Duyne RP. 2013. Creating, characterizing, and controlling chemistry with SERS hot spots. Phys Chem Chem Phys. 15(1):21-36.

[10] Prochazka M. 2016. Surface-Enhanced Raman 
Spectroscopy_Chapter 3_Basics of Surface-Enhanced Raman Scattering (SERS) [Internet]. 2016.

[11] Turkevich J, Stevenson PC, Hillier J. 1951. A study of the nucleation and growth processes in the synthesis of colloidal gold // Discuss. Faraday Soc. Vol 11 P. 11:055.

[12] Frens G. 1973. Controlled Nucleation for the Regulation of the Particle Size in Monodisperse Gold Suspensions. Nat Phys Sci. 241(105):20-2. [13] Lee PC, Meisel D. 1982. Adsorption and surfaceenhanced Raman of dyes on silver and gold sols. J Phys Chem. 86(17):3391-5.

[14] Pelaz B, Grazu V, Ibarra A, Magen C, Del Pino P, De La Fuente JM. 2012. Tailoring the synthesis and heating ability of gold nanoprisms for bioapplications.

Langmuir. 28(24):8965-70.

[15]Paramelle D, Sadovoy A, Gorelik S, Free P, Hobley J, Fernig DG. 2014. A rapid method to estimate the concentration of citrate capped silver nanoparticles from UV-visible light spectra. Analyst. 139(19):4855-61.
[16] Haiss W, Thanh NTK, Aveyard J, Fernig DG. 2007. Determination of size and concentration of gold nanoparticles from UV-Vis spectra. Anal Chem. 79(11):4215-21.

[17] Liu X, Atwater M, Wang J, Huo Q. 2007. Extinction coefficient of gold nanoparticles with different sizes and different capping ligands. Colloids Surfaces B Biointerfaces. 58(1):3-7. [18]Jaworska A, Malek K, Marzec KM, Baranska M. 2012. Nicotinamide and trigonelline studied with surface-enhanced FT-Raman spectroscopy. Vib Spectrosc. 63:469-76.

[19] Eravuchira PJ, El-Abassy RM, Deshpande S, Matei MF, Mishra S, Tandon P, et al. 2012. Raman spectroscopic characterization of different regioisomers of monoacyl and diacyl chlorogenic acid. Vib Spectrosc. 61:10-6.

[20] Long GL, Winefordner JD. 1983. Limit of Detection. Anal Chem. 55(7):A712-24. 